\title{
Upregulation of syncytin-I promotes invasion and metastasis by activating epithelial-mesenchymal transition-related pathway in endometrial
} carcinoma

This article was published in the following Dove Press journal:

OncoTargets and Therapy

\author{
Changmin Liu, ${ }^{1, *}$ Jiqin $\mathrm{Xu},{ }^{2, *}$ \\ Feifei Wen, ${ }^{3}$ Fangfang Yang,' \\ Xiaoming Li, ${ }^{4}$ Dianzhong \\ Geng,' Lei Li,' Jiming Chen, ${ }^{5}$ \\ Jing Zheng ${ }^{6}$ \\ 'Department of Oncology, Binzhou \\ Medical University Hospital, Binzhou \\ 256603, Shandong, China; ${ }^{2}$ Department \\ of Obstetrics and Gynecology, Shuyang \\ People's Hospital, Affiliated to Xuzhou \\ Medical University, Jiangsu, China; \\ ${ }^{3}$ Department of Pathology, Binzhou \\ Medical University Hospital, Binzhou \\ 256603, Shandong, China; ${ }^{4}$ Department of \\ Hepatobiliary Surgery, Shandong Provincial \\ Hospital Affiliated to Shandong University, \\ Jinan, Shandong, China; ${ }^{5}$ Department of \\ Obstetrics and Gynecology, The Affiliated \\ Changzhou No 2 People's Hospital of \\ Nanjing Medical University, Changzhou \\ 213000, Jiangsu, China; ' Department of \\ Clinical Medicine Laboratory, Binzhou \\ Medical University Hospital, Binzhou \\ 256603, Shandong, China \\ *These authors contributed equally \\ to this work
}

Background: Endometrial carcinoma (EC) is the most common and lethal malignancy worldwide. Syncytin-1 is expressed in multiple types of cancer. However, the expression pattern and potential mechanism of syncytin-1 and its clinical significance in EC remain unclear.

Materials and methods: We analyzed 130 primary EC specimens from Binzhou Medical University to investigate the clinical role of syncytin-1 in EC by using different advanced pathological stages of EC tissues. Kaplan-Meier analysis was used to measure the overall survival of EC patients. Syncytin-1 expression was analyzed by Western blot assays in HECCL-1 and RL-95-2 cells. Cell proliferation, cycle, migration, and invasion abilities were detected by cell counting kit-8, flow cytometry, and transwell assays. AKT and epithelial-mesenchymal transition (EMT)-related genes were assessed by Western blot assays in HECCL-1 and RL-95-2 cells.

Results: Syncytin-1 was upregulated in EC tissues and cells and was related to clinical stages, expression of ER, Ki-67, and overall survival of EC. Functional research revealed that overexpression of syncytin-1 can promote cell proliferation, cell cycle progression, and the migration and invasion of EC cells. Suppression of syncytin-1 expression also inhibited cell proliferation and apoptosis in vitro. The expression of syncytin-1 substantially improved the expression levels of EMT-related genes (vimentin, E-cadherin, slug, and ZEB1) but significantly decreased those of epithelial markers (N-cadherin and snail). In addition, we found that syncytin-1 was not correlated with AKT-related genes (total-AKT, p-AKT, and vinculin).

Conclusion: Our results suggested that syncytin-1 may promote aggressive behavior and can serve as a novel prognostic biomarker for EC. Our study provides new insights into the regulatory mechanism of EMT signaling.

Keywords: syncytin-1, epithelial-mesenchymal transition, endometrial carcinoma

\section{Introduction}

Department of Obstetrics and Gynecology,

The Affiliated Changzhou No 2 People's

Hospital of Nanjing Medical University,

Changzhou 213000, Jiangsu, China

Email cjming@।26.com

Jing Zheng

Department of Clinical Medicine Laboratory, Binzhou Medical University Hospital,

The Yellow River two Road No 66I, Binzhou 256603, Shandong, China

Tel +86543325 878।

Email zhengjing2003@I26.com
Endometrial carcinoma (EC) is one of the most common malignancies and ranks fourth in female cancers. ${ }^{1}$ In China, the morbidity and mortality of EC increase with population size, ${ }^{2}$ thus prompting an urgent need to develop effective treatments and identify novel therapeutic targets for EC. Multiple factors such as clinical factors (surgical-pathologic staging, depth of myometrial invasion, and adjunctive therapy) and biological factors (steroid receptors, growth factors, oncogenes, and suppressor genes) affect the therapeutic treatment of EC. ${ }^{3-5}$ Immunomodulatory disorders, such as persistent inflammation, play a significant role in its promotion. ${ }^{6-8}$ Anti-inflammatory 
molecules may therefore be potential candidates for cancer treatment.

Syncytin-1 belongs to a family of endogenous retroviruses and is the trace left by HERVW1 after infecting human germ cells. ${ }^{9-11}$ Introduction of syncytin-1 in a cell line prevents retrovirus infection, ${ }^{12-15}$ suggesting that the co-expression of syncytin-1 and its receptor blocks either of the two. In humans, syncytin-1 is most abundantly expressed in the placental trophoblast lineage and may be used as a diagnostic tool in evaluating reproductive potential. ${ }^{16-19}$ In addition, syncytin-1 exhibits antiapoptotic activity in both knockdown and overexpression models in vitro. ${ }^{20-22}$

The expression of syncytin- 1 is activated and upregulated in various malignancies, including breast cancer, ovarian cancer, colorectal cancer, kidney cancer, leukemia, lymphoma, and EC. ${ }^{23-27}$ Although the expression level of syncytin-1 varies in different tumors, the pathological significance of its non-fusion activity remains to be studied. However, current evidence suggests that measuring syncytin-1 expression levels in cancer tissues may have some prognostic values for certain tumor types and stages. ${ }^{28}$

In this study, we verified the roles of syncytin-1 in EC cell proliferation, migration, and invasion and identified the mechanisms of syncytin-1 in EC, such as its relationship with epithelial-mesenchymal transition (EMT)-related pathway genes. We found that syncytin-1 may be a novel therapeutic target for the treatment of EC.

\section{Materials and methods Immunohistochemistry}

A total of 130 archived clinical EC specimens and 12 normal endometrial tissues were obtained at the Binzhou Medical University Hospital of China between January 2012 and January 2015 with patient anonymity and informed consent. This study was approved by the Ethical Review Committee of Binzhou Medical University Hospital. Participants provided written informed consent, and the study was performed according to the principles of the Declaration of Helsinki. For deparaffinization and rehydration, the tissue array was sequentially treated with xylene (20 minutes $\times 3$ ), $100 \%$ ethanol ( 5 minutes $\times 2$ ), $95 \%$ ethanol ( 5 minutes $\times 2$ ), $80 \%$ ethanol ( 5 minutes $\times 1$ ), $70 \%$ ethanol $(5$ minutes $\times 1$ ), and distilled water $(5$ minutes $\times 2$ ). Rabbit polyclonal antisyncytin was also used (1:50; Novus, Littleton, CO, USA). Following extensive washing, secondary antibody (1:500, IHC-101; Bethyl Laboratories, Inc., Montgomery, TX, USA) was applied. Color development was conducted using diaminobenzidine tetrahydrochloride (Bethyl Laboratories,
Inc.). Tissue slides were counterstained with hematoxylin (Santa Cruz Biotechnology Inc., Dallas, TX, USA). Semiquantitative assessment was performed by scoring the staining intensity as $0,1,2$, or 3 , which corresponds to negative, weak, moderate, or strong staining intensity, respectively. The patients were divided into low expression group (negative and weak) and high expression group (moderate and strong) according to immunohistochemistry. The results were blinded by two pathologists.

\section{Cell culture}

Human EC cell lines KLE, RL-95-2, HECCL-1, HEC-1-A, and JEC and immortalized endometrial epithelial EM-E6/ E7-TERT were obtained from the Shanghai Cell Bank at the Chinese Academy of Sciences (Shanghai, China), and their use was approved by the Ethical Review Committee of Binzhou Medical University Hospital. All cell lines were cultured in RPMI-1640 medium (Hyclone, Logan, UT, USA) supplemented with 10\% FBS (Thermo Fisher Scientific, Waltham, MA, USA). The cells were cultured at $37^{\circ} \mathrm{C}$ in a humidified atmosphere containing $5 \% \mathrm{CO}_{2}$. Transfection was performed using Lipofectamine 2000 Reagent (Thermo Fisher Scientific) following the manufacturer's instructions.

\section{Quantitative real-time PCR (qRT-PCR) analysis}

Total RNA was extracted from the tissues using Trizol reagent (TaKaRa, Shiga, Japan) following the manufacturer's instructions. cDNA was synthesized from $1 \mu \mathrm{g}$ of total RNA using the transcriptor first strand cDNA synthesis kit (Hoffman-La Roche Ltd., Basel, Switzerland). Realtime PCR was performed by Faststart Universal SYBR Green Master (Hoffman-La Roche Ltd.). The primers were synthesized by Sangon Biotech Co., Ltd. (Shanghai, China). The primers were as follows $\left(5^{\prime}-3^{\prime}\right)$ : syncytin-1 forward primer: GGAGGAGATGTGGCACCATT, reverse primer: CCTTCCCACCACAGAAGACC. $\beta$-actin forward primer: TGCTGTCCCTGTATGCCTCT, and reverse primer: AGGTCTTTACGGATGTCAACG. Data were analyzed by the method of $2^{-\Delta \Delta \mathrm{Ct}}$.

\section{Western blotting}

For Western blot analysis, approximately $10 \mu \mathrm{g}$ of proteins were loaded and separated on the BioRad mini gel system (Bio-Rad Laboratories Inc., Hercules, CA, USA). Protein expression of syncytin-1 and GAPDH was detected by incubating with antibodies anti-syncytin- 1 and anti-GAPDH (ZSGB-BIO, Beijing, China) at a dilution of 1:1,000 
overnight at $4^{\circ} \mathrm{C}$ in primary antibodies. The membranes were then incubated for $2 \mathrm{~h}$ at room temperature in HRP-labeled secondary antibody (Pierce, Rockford, IL, USA) (1:1,000). The bands were visualized using colorimetric detection and exposure to autoradiography film.

\section{Cell cycle analysis}

RL-95-2 cell was seeded in a six-well plate, incubated for 24 hours, and then collected for cell cycle analysis as previously described.

\section{Plasmid construction and transfection}

The complete sequence of human syncytin-1 gene was synthesized and inserted into a pcDNA3.1 (+) vector (GenePharma, Shanghai, China) to construct the pcDNA3.1Syncytin-1 expression vector. RL-95-2 cells $\left(5 \times 10^{5}\right.$ cells) at the logarithmic phase were inoculated in six-well plates and incubated for 12 hours. The cells were then transfected with pcDNA3.1 and pcDNA3.1-syncytin-1 plasmid by using Lipofectamine 2000 reagent (cat. no 11668-019; Thermo Fisher Scientific) and Opti-MEM-reduced serum medium (Thermo Fisher Scientific) according to the manufacturer's instructions until the cell fusion rate reached $80 \%$.

\section{Cell counting kit (CCK)-8 assay}

HECCL-1 and RL-95-2 cells seeded in 96-well plates $(2,000$ cells/well) were allowed to attach overnight and then left untreated or treated with Au-NPs $(5,10,20,40$, 50 , or $60 \mathrm{~nm}$ ) for 24 hours. Assay reagent (cat. no KGA317; CCK-8; Kaiji, Nanjing, China) was subsequently added to each well, followed by an incubation for 1, 2, 3, and 4 hours. Absorbance values at $450 \mathrm{~nm}$ were recorded using a microplate reader (iMark Microplate Absorbance Reader; Bio-Rad Laboratories, Inc.). The results from each of the four time points were averaged, and cell viability was calculated as a percentage of the untreated control. Each experiment was performed in triplicate, and the experiment was repeated three times.

\section{Transwell assay}

Cell migration and invasion were confirmed by detecting the ability of cells to move to another place. The cells at the logarithmic phase were digested, washed with PBS twice, and suspended in RPMI-1640 medium without FBS. The concentration was adjusted to $2 \times 10^{5}$ cells $/ \mathrm{mL}$. Cell suspensions $(100 \mu \mathrm{L})$ were added to the polycarbonate membrane of the upper chamber with (for the invasion assay) or without (for the migration assay) Matrigel (BD
Biosciences, San Jose, CA, USA). The bottom chamber was filled with complete medium $(500 \mu \mathrm{L})$. The cells were incubated at $37^{\circ} \mathrm{C}$ for 24 hours. The cells on the bottom of the coated Transwell chamber were washed twice, fixed with 4\% paraformaldehyde (CatP6148) for 30 minutes, and stained with $0.1 \%$ crystal violet (no C3886-100G0; SigmaAldrich Co., St Louis, MO, USA) for 15 minutes at room temperature. After drying, the number of migrated or invaded cells was examined from five randomly selected fields under a microscope at a magnification of $\times 100$.

\section{Statistical analyses}

Statistical analyses of experimental data were conducted with Prism 5.0 (GraphPad Software, Inc., La Jolla, CA, USA). All results are presented as the mean $\pm \mathrm{SD}$ from at least three independent experiments. $P<0.05$ was considered statistically significant.

\section{Ethics statement}

This study was approved by the Ethics Committee of the Binzhou Medical College Affiliated Hospital (No 2018024-03).

\section{Results}

\section{Validation of syncytin-I expression in EC and noncancerous tissues}

To validate the profile and characterize the expression pattern of syncytin-1 in EC, we collected three pairs of fresh EC and adjacent normal tissues. RT-PCR showed that syncytin-1 mRNA level was significantly increased in EC compared with the adjacent normal tissues in samples 1 and $2(P<0.001)$ but not in sample $3(P>0.05)$ (Figure 1A). We believe that the possible reason is the genetic heterogeneity for the different samples.

\section{Correlation analysis of syncytin- I expression and clinicopathological parameters of EC}

A total of 130 archived clinical EC specimens and 37 normal endometrial tissues were examined by immunohistochemistry analysis to assess the relationship between the expression of syncytin-1 and the progression of EC (Table 1). The results showed that syncytin-1 was mainly localized in the cytoplasm (Figure 1B). Syncytin-1 was highly expressed in 15 out of 37 (40.54\%) cases of human normal endometrial tissues and in 83 out of 130 (63.85\%) cases of EC with significant difference $\left(\chi^{2}=6.452, P=0.011\right)$. Statistical analyses were performed to explore the correlation 
A

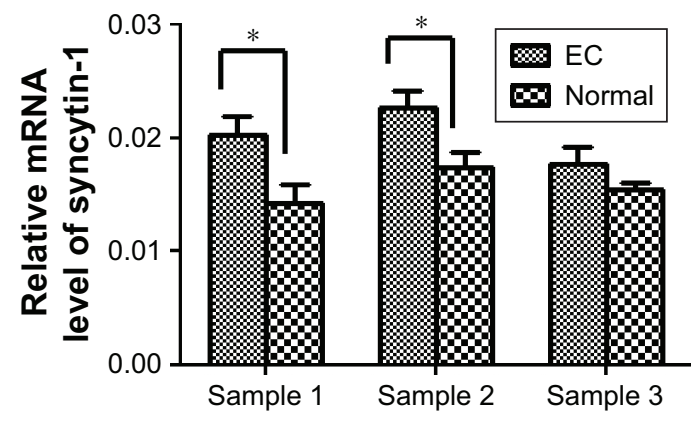

B

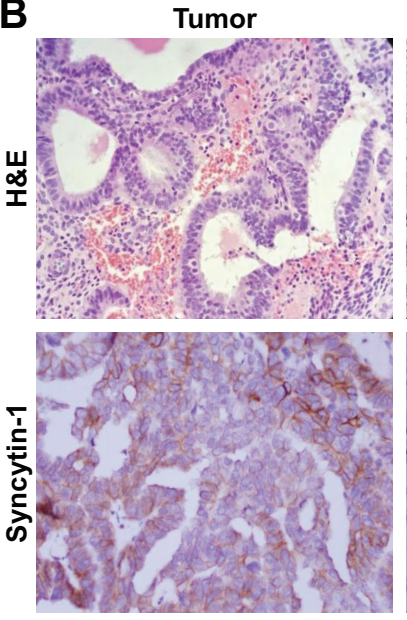

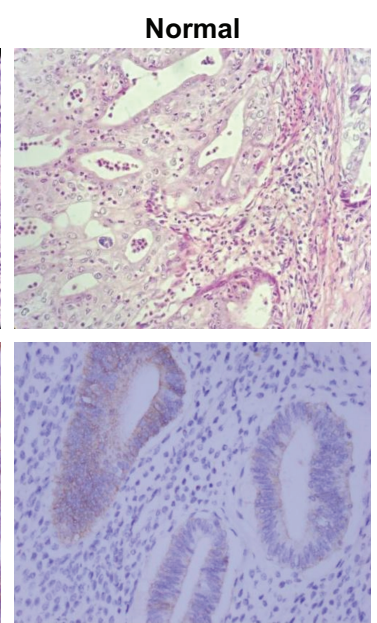

C

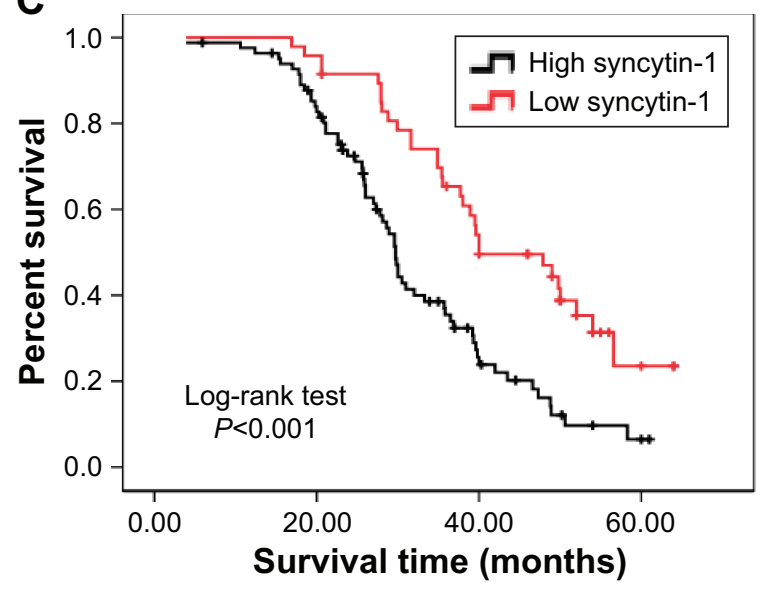

Figure I Relative syncytin-I expression in EC and the relationship with the overall survival of EC.

Notes: (A) Relative expression of syncytin-I among tumor and peritumor tissues using qRT-PCR ( $n=3$, *P $<0.00 I$ ). (B) Syncytin-I protein localized mostly on the membrane and in cytoplasm, with low staining observed in nucleus for both normal and carcinoma tissues. Reduced level of syncytin-I expression found in normal tissues compared with that in EC tissues. Representative syncytin-I and H\&E staining. Scale bar $=50 \mu$ m, magnification: $400 \times$. (C) Kaplan-Meier survival curves showed that patients with EC and high expression of syncytin-I have poorer prognosis than those with low expression $(P<0.00 \mathrm{I})$.

Abbreviations: EC, endometrial carcinoma; H\&E, hematoxylin and eosin; qRT-PCT, quantitative real-time PCR.

between syncytin-1 expression and the clinical characteristics of EC as detected by immunohistochemical staining. The result of chi-squared test showed that syncytin-1 expression was positively correlated with tumor FIGO stages ( $P=0.001)$, expression of ER $(P=0.018)$, and expression of Ki-67 $(P=0.037)$ (Table 2$)$, thereby indicating that these clinical features are correlated with syncytin-1 expression.

\section{Syncytin-I expression was related to the overall survival of EC}

Kaplan-Meier analysis was used to assess the overall survival according to syncytin- 1 expression and to further explore the correlation between syncytin-1 expression and EC survival. The results showed that the mean survival time was 39.18 \pm 4.36 months in patients with EC and high syncytin-1 expression and 51.12 \pm 2.78 months in those with low expression of syncytin-1. The 5-year cumulative survival rate was $32.8 \%$ in patients with EC and high syncytin-1 expression and $60.7 \%$ in those with low expression of syncytin-1. Patients with EC and high expression of syncytin-1 have a poor prognosis (Figure $1 \mathrm{C}, \chi^{2}=14.512, P<0.001$ ). Therefore, syncytin- 1 expression is associated with survival and prognosis.

\section{Expression of syncytin-I in EC cell lines}

We evaluated the expression of syncytin-1 in EC cell lines KLE, RL-95-2, HECCL-1, HEC-1-A, and JEC and immortalized endometrial epithelial EM-E6/E7-TERT. Western blot analysis showed that the expression of syncytin-1 protein was markedly increased in KLE and RL-95-2 but was low in other EC cell lines. We believe that this phenomenon is the result of the selective expression of genes that control traits in time and space. The expression of syncytin-1 in all EC cell lines was significantly higher than that in normal endometrial 
Table I Clinicopathological characteristics of patient samples

\begin{tabular}{l|l}
\hline Characteristics & Number of cases (\%) \\
\hline $\begin{array}{l}\text { Age (years) } \\
\leq 50\end{array}$ & $5 \mathrm{I}(39.2)$ \\
$>50$ & $79(60.8)$ \\
FIGO stage & $71(54.6)$ \\
I & $38(29.2)$ \\
II & $21(16.2)$ \\
III & $0(0)$ \\
IV & $109(83.8)$ \\
Pathological differentiation & $21(16.2)$ \\
Well & \\
Poor & $83(63.8)$ \\
Expression of syncytin-I & $47(36.2)$ \\
High & $18(13.8)$ \\
Low & $112(86.2)$ \\
Expression of ER & \\
Negative & $13(10.0)$ \\
Positive & $117(90.0)$ \\
Expression of PR & $17(13.1)$ \\
Negative & $113(86.9)$ \\
Positive & \\
Expression of Ki-67 & \\
$\leq 20 \%$ & \\
$>20 \%$ &
\end{tabular}

Table 2 Clinicopathological characteristics of patient samples and expression of syncytin-I in EC and correlation between syncytin-I expression and clinicopathological characteristics of EC patients

\begin{tabular}{|c|c|c|c|c|}
\hline \multirow[t]{2}{*}{ Characteristics } & \multirow[t]{2}{*}{ Total } & \multicolumn{2}{|c|}{ Syncytin-I } & \multirow{2}{*}{$\begin{array}{l}\text { Chi-squared } \\
\text { test } \\
P \text {-value }\end{array}$} \\
\hline & & High & Low & \\
\hline \multicolumn{5}{|l|}{ Age (years) } \\
\hline$\leq 50$ & 51 & 33 & 18 & 0.027 \\
\hline$>50$ & 79 & 50 & 29 & 0.870 \\
\hline \multicolumn{5}{|l|}{ FIGO stage } \\
\hline I & 71 & 35 & 36 & \\
\hline II & 38 & 31 & 7 & $|4.35|$ \\
\hline III & 21 & 17 & 4 & 0.001 \\
\hline IV & 0 & 0 & 0 & \\
\hline \multicolumn{5}{|l|}{$\begin{array}{l}\text { Pathological } \\
\text { differentiation }\end{array}$} \\
\hline Well & 109 & 69 & 40 & 0.086 \\
\hline Poor & 21 & 14 & 7 & 0.769 \\
\hline \multicolumn{5}{|l|}{ Expression of ER } \\
\hline Negative & 18 & 7 & 11 & 5.638 \\
\hline Positive & 112 & 76 & 36 & 0.018 \\
\hline \multicolumn{5}{|l|}{ Expression of PR } \\
\hline Negative & 13 & 10 & 3 & 1.070 \\
\hline Positive & 117 & 73 & 44 & 0.301 \\
\hline \multicolumn{5}{|l|}{ Expression of Ki-67 } \\
\hline$\leq 20 \%$ & 17 & 7 & 10 & 4.354 \\
\hline$>20 \%$ & 113 & 76 & 37 & 0.037 \\
\hline \multicolumn{5}{|l|}{ Tissue } \\
\hline EC & 130 & 83 & 47 & 6.452 \\
\hline Normal & 37 & 15 & 22 & 0.011 \\
\hline
\end{tabular}

Abbreviation: EC, endometrial carcinoma. epithelial cell line EM-E6/E7-TERT. The expression of endogenous syncytin-1 in RL-95-2 cells was significantly higher than that in other EC cells, in which HECCL-1 cells was the lowest (Figure 2A and B). This result provides the basis and premise for the subsequent exogenously highly expressed adenovirus syncytin-1 or sh-syncytin-1 as a tool to study the effects of syncytin-1 on the function of both HECCL-1 and RL-95-2 cell lines.

\section{Establishment of a stable syncytin-I- overexpressing cell line}

The fragment of human syncytin-1 was successfully cloned to explore its function and mechanism in EC. The fragment was subcloned into the lentiviral vector and verified by DNA sequencing. The expression of syncytin-1 protein in HECCL-1 cells was effectively increased after 48 hours of adenovirus infection. The successfully verified virus provides a reliable tool for subsequent experiments. Significant inhibition of syncytin-1 expression was found in RL-95-2 cell line, suggesting that the RNA interference sequence can target the inhibition of syncytin-1 expression in EC cell lines RL-95-2 with the use of reliable tools (Figure 2C and D).

\section{Syncytin-I promotes EC cell proliferation}

The viability of HECCL-1 and RL-95-2 cells was assessed at different time points (days 1-7) using CCK-8 assay and plate cloning experiment to explore the effect of syncytin-1 on EC cell growth. Compared with the control group, overexpression of syncytin-1 significantly promoted HECCL-1 tumor cell growth $(P<0.01$, Figure $3 \mathrm{~A}-\mathrm{C})$. Compared with the shNC group, shSyncytin-1 significantly suppressed proliferation in RL-95-2 tumor cell growth $(P<0.05$, Figure 3D-F).

\section{Syncytin-I promotes EC cell migration and invasion}

Considering that syncytin-1 promotes EC cell proliferation, we further assessed the effect of syncytin-1 on migration and invasion. Data showed that overexpression of syncytin-1 markedly promoted the migration and invasion capacities of HECCL-1 ( $P<0.01, P<0.001$, Figure 4A-D), whereas the shSyncytin-1 in RL-95-2 cells markedly inhibited the migration and invasion capacities $(P<0.001$, Figure $4 \mathrm{E}-\mathrm{H})$.

\section{Syncytin-I promotes G2/M phase transition in the cell cycle of EC}

Cell cycle was examined using shSyncytin-1 to specifically interfere with the syncytin-1 sequence of EC cell line RL-95-2. Compared with sh-NC, shSyncytin-1 
A

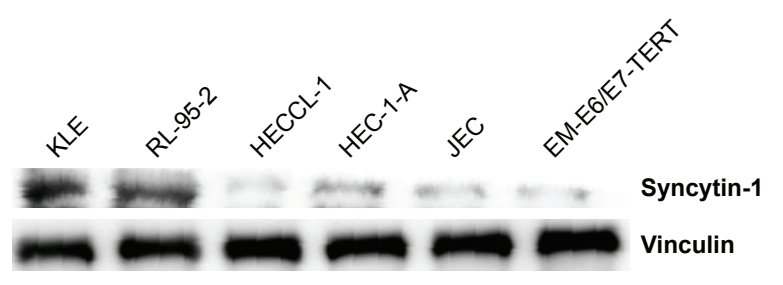

B

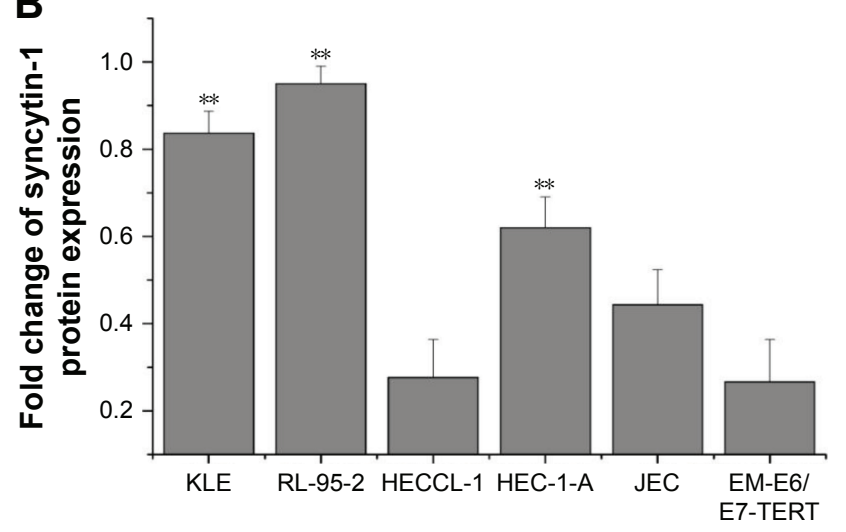

C

HECCL-1

$+\quad-\mathrm{pCDH}$

- + Flag-syncytin-1

Flag

Vinculin

D

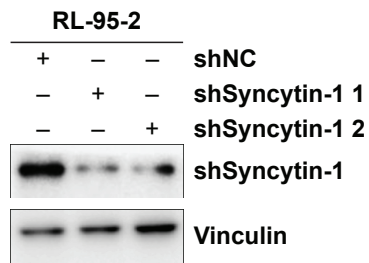

Figure 2 Expression of syncytin-I in EC cell lines by Western blot.

Notes: (A and B) Syncytin-I is highly expressed in KLE and RL-95-2 cell lines. Its expression in all EC cell lines was higher than that in normal endometrial epithelial cell line EM-E6/E7-TERT. $* * P<0.05$. (C) Overexpression of syncytin-I in HECCL-I cell line, detection of tag flag. (D) shSyncytin- I expression in RL-95-2 cell lines.

Abbreviation: EC, endometrial carcinoma.

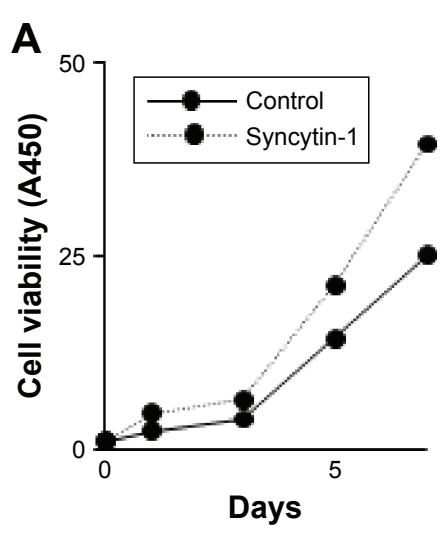

D

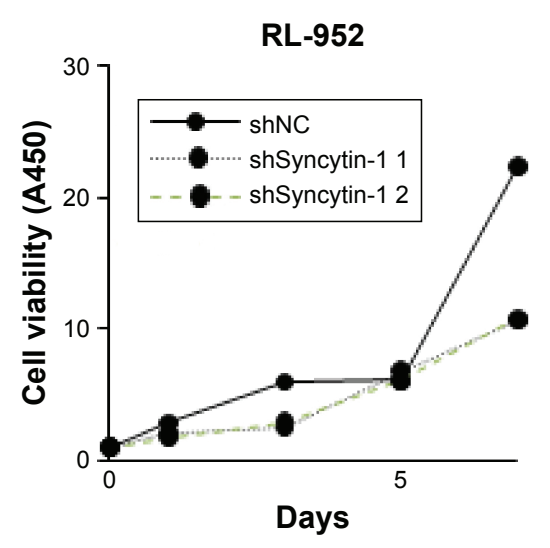

B

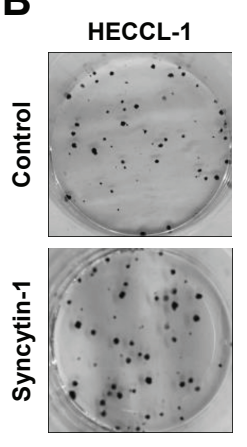

E

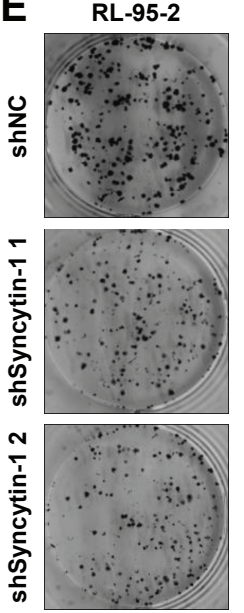

C

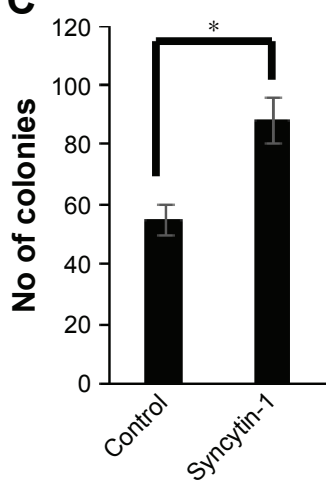

F

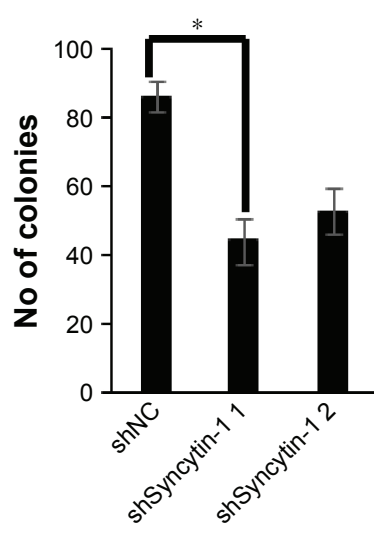

Figure 3 CCK-8 and plate cloning experiment detect the growth ability of cell line.

Notes: (A-C) Comparison with the control group using a CCK-8 assay and late cloning experiment showed that overexpression of syncytin-I significantly promotes HECCL-I tumor cell growth. $* P<0.0 I$. (D-F) Comparison with the shNC group using a CCK-8 assay and plate cloning experiment confirmed that shSyncytin-I significantly suppresses the proliferation in RL-95-2 tumor cell growth. $* P<0.01$. 

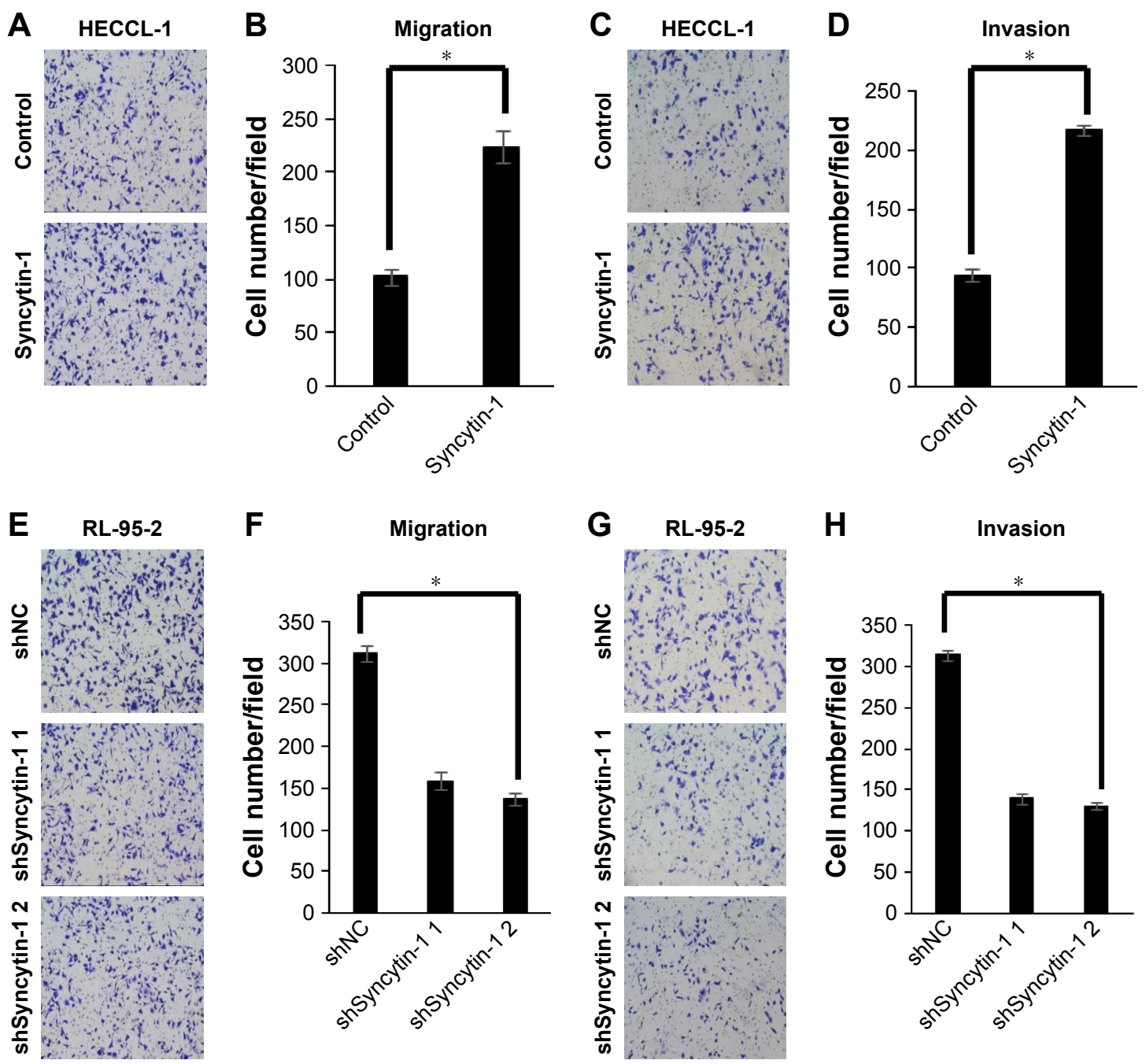

Figure 4 Syncytin-I promotes endometrial cancer cell migration and invasion.

Notes: Migration (A, B) and invasion (C, D) abilities of the overexpressed syncytin-I in HECCL-I tumor cells were measured by transwell assays and migration and invasion were promoted than control $(* P<0.00 I)$. Migration $(\mathbf{E}, \mathbf{F})$ and invasion $(\mathbf{G}, \mathbf{H})$ abilities of shSyncytin-I in RL-95-2 cells were measured by transwell assays and migration and invasion were suppressed than $\mathrm{NC}$ group $(* P<0.01)$.

can significantly increase the proportion of $\mathrm{G} 2 / \mathrm{M}$ (from $11.29 \%$ to $37.62 \%$ ) and the percentage of S-phase cells (from $16.68 \%$ to $42.56 \%$ ) (Figure 5). This finding showed that inhibiting the expression of syncytin-1 promotes cell stagnation in the RL-95-2 cell lines. Syncytin-1 stimulates the change in G2-M phase, thereby inducing many paralyzed cells to enter mitosis.

\section{Effects of syncytin-I on the expression of EMT-related proteins}

To explore the mechanism underlying the cell cycle arrest, we detected the expression levels of the cell cycle-related proteins by Western blot analysis. The results showed that overexpression of syncytin-1 can significantly regulate the changes in EMT-related molecules mainly by promoting the expression of vimentin, E-cadherin, slug, and ZEB1 and by inhibiting the expression of $\mathrm{N}$-cadherin and snail in
HECCL-1 cells. shSyncytin-1 could decrease the expression of vimentin, E-cadherin, slug, and ZEB1 in RL-95-2 cells (Figure 6).

\section{Effects of syncytin-I on the expression of AKT-related proteins}

The association between syncytin-1 and AKT was investigated through Western blot to clarify the role of the former in the progression of human EC and to elucidate the underlying mechanism. Overexpression of syncytin-1 and shSyncytin-1 was not correlated with the AKT-related genes (total-AKT, p-AKT, and vinculin) in HECCL-1 and RL-95-2 cells (Figure 7).

\section{Discussion}

Syncytin-1 is a common protein in human reproductive system envelope and is located in the first trimester 


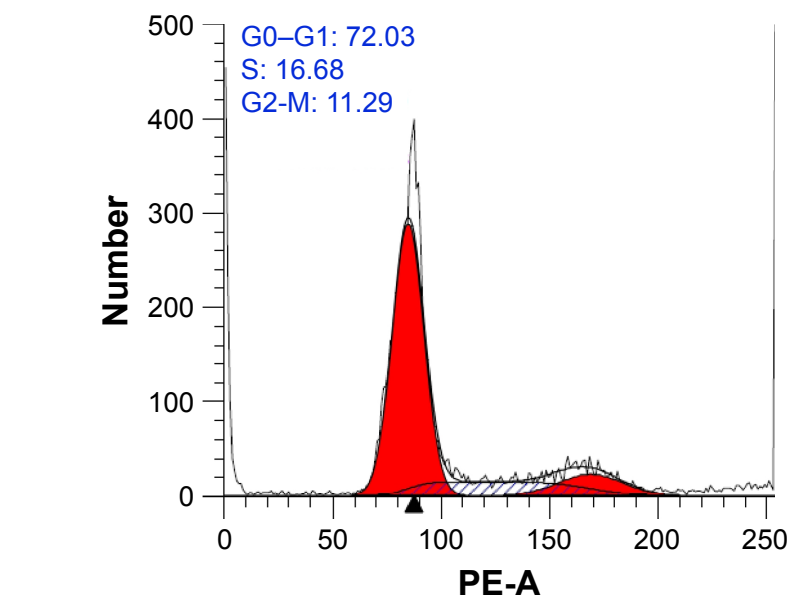

shSyncytin-1

shNC
0

100

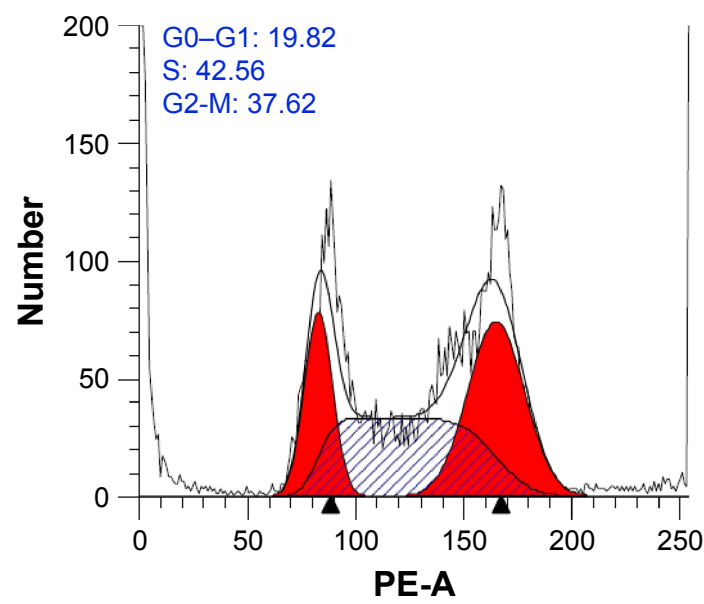

100

0

Dip G0-G1 — Dip G2-M Z Dip S

Figure 5 Apoptosis and proliferation changes by shSyncytin-I in RL-95-2 cells.

trophoblast. ${ }^{29,30}$ Its abnormal expression may lead to intrauterine growth retardation, pathological embryonic, tumor, and multiple sclerosis. This protein has critical roles in many diseases. ${ }^{12,31-40}$ However, the exact regulatory mechanism of syncytin-1 in EC is poorly understood. To solve this problem, we transfected the expression of syncytin-1 in EC cells by pc-Syncytin-1 and shSyncytin-1 and analyzed the effect of the abnormal expression of syncytin-1 on cell proliferation and cell metastasis.

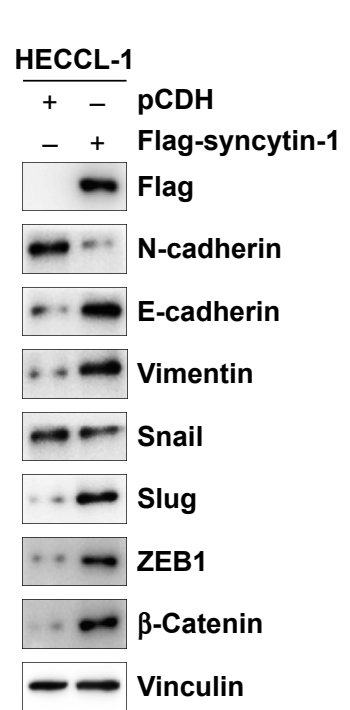

Figure 6 Effects of syncytin-I on the expression of specific proteins. Notes: (A) Western blot analysis showed that overexpression of syncytin-I could increase the expression of vimentin, E-cadherin, slug, and ZEBI protein. (B) shSyncytin-I could decrease the expression of vimentin, E-cadherin, slug, and ZEBI protein.
According to our clinical and biochemical data, syncytin-1 is upregulated in EC tissues and cell lines. Further studies found that syncytin-1 can promote cell proliferation, migration, and invasion and the change in G2-M phase. These findings suggest that syncytin-1 may be a regulatory gene for EC. EMT is an effective way for epithelial cells to acquire migratory capacity ${ }^{41-48}$ and has become an important pathway for invasion and metastasis of epithelial cell carcinoma. Various studies showed that the development of EMT is accompanied with changes in markers, including E-cadherin, N-cadherin, and vimentin. In the present study, syncytin-1 significantly promotes the expression of vimentin, E-cadherin, slug, and ZEB1 protein but inhibits the expression of $\mathrm{N}$-cadherin and snail. This finding indicates a new molecular mechanism by which syncytin-1 regulates cell migration in EC cells.
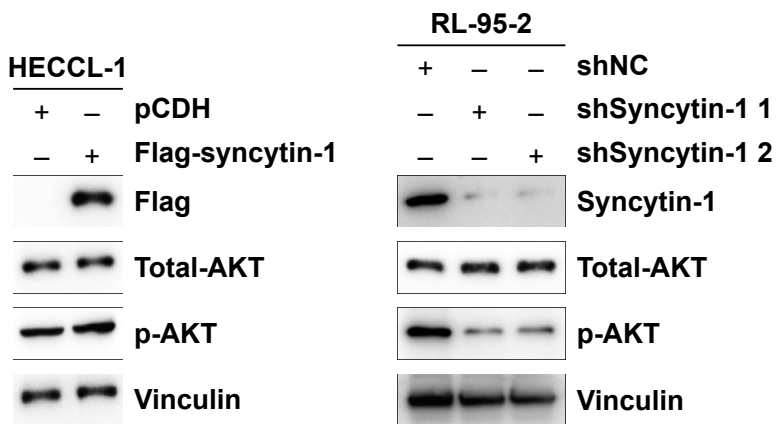

Figure 7 Effects of syncytin-I on the expression of AKT-related proteins. Notes: (A) Western blot analysis showed that overexpression of syncytin-I could not increase the expression of total-AKT, P-AKT, and vinculin protein. (B) AKT-related proteins were detected by Western blotting after shSyncytin-I treatment. 


\section{Conclusion}

Syncytin-1 plays as a tumor promoter in the occurrence and carcinogenesis of EC. Understanding the precise function of syncytin-1 in complex networks may provide further insights into the progression of EC and offers a promising therapeutic target for the treatment of this disease.

\section{Acknowledgment}

This research was supported by grants from the Medicine and Health and Scientific Development Programme of Shandong Province (2015WSB30011) and the Scientific Development Programme of Binzhou Medical University (BY2014KJ45, BY2017KJ09).

\section{Disclosure}

The authors report no conflict of interest in this work.

\section{References}

1. Siegel RL, Miller KD, Jemal A. Cancer statistics, 2018. CA Cancer J Clin. 2018;68(1):7-30.

2. Liu SP, Cheng XW, Tian X, Zhang Q, Cui HY, Hua KQ. Laparoscopic surgery for endometrial cancer in aged patients: experience from a tertiary referral center in Eastern China. J Cancer Res Ther. 2017;13(5): 761-766.

3. Dessai SB, Adrash D, Geetha M, et al. Pattern of care in operable endometrial cancer treated at a rural-based tertiary care cancer center. Indian J Cancer. 2016;53(3):416-419.

4. Kulhan M, Kulhan G, Nayki U, et al. Assessment of clinicopathological features, evaluation of treatment, and prognosis of clear cell and serous papillary endometrial carcinoma. Ginekol Pol. 2016;87(8): $570-574$

5. Inoue O, Hamatani T, Susumu N, et al. Factors affecting pregnancy outcomes in young women treated with fertility-preserving therapy for well-differentiated endometrial cancer or atypical endometrial hyperplasia. Reprod Biol Endocrinol. 2016;14:2.

6. Enninga EA, Holtan SG, Creedon DJ, et al. Immunomodulatory effects of sex hormones: requirements for pregnancy and relevance in melanoma. Mayo Clin Proc. 2014;89(4):520-535.

7. Sanderson PA, Critchley HO, Williams AR, Arends MJ, Saunders PT. New concepts for an old problem: the diagnosis of endometrial hyperplasia. Hum Reprod Update. 2017;23(2):232-254.

8. Trabert B, Eldridge RC, Pfeiffer RM, et al. Prediagnostic circulating inflammation markers and endometrial cancer risk in the prostate, lung, colorectal and ovarian cancer (PLCO) screening trial. Int $J$ Cancer. 2017;140(3):600-610.

9. Grandi N, Tramontano E. HERV envelope proteins: physiological role and pathogenic potential in cancer and autoimmunity. Front Microbiol. 2018;9:462.

10. Makaroun SP, Himes KP. Differential methylation of syncytin-1 and 2 distinguishes fetal growth restriction from physiologic small for gestational age. AJP Rep. 2018;8(1):e18-e24.

11. Grandi N, Cadeddu M, Blomberg J, Mayer J, Tramontano E. HERV-W group evolutionary history in non-human primates: characterization of ERV-W orthologs in Catarrhini and related ERV groups in Platyrrhini. BMC Evol Biol. 2018;18(1):6.
12. Grandi N, Tramontano E. Human Endogenous Retrovirus (HERV-W) integrations and their mobilization by L1 machinery: contribution to the human transcriptome and impact on the host physiopathology. Viruses. 2017;9(7):E162.

13. Machnik G, Klimacka-Nawrot E, Sypniewski D, et al. Porcine endogenous retrovirus (PERV) infection of HEK-293 cell line alters expression of human endogenous retrovirus (HERV-W) sequences. Folia Biol. 2014;60(1):35-46.

14. Li F, Nellåker C, Sabunciyan S, et al. Transcriptional derepression of the ERVWE1 locus following influenza A virus infection. J Virol. 2014; 88(8):4328-4337.

15. Nellåker C, Yao Y, Jones-Brando L, Mallet F, Yolken RH, Karlsson H. Transactivation of elements in the human endogenous retrovirus $\mathrm{W}$ family by viral infection. Retrovirology. 2006;3:44.

16. Wang X, Huang J, Zhu F. Human Endogenous Retroviral Envelope Protein Syncytin-1 and Inflammatory Abnormalities in Neuropsychological Diseases. Front Psychiatry. 2018;9:422.

17. Grandi N, Tramontano E. HERV Envelope Proteins: Physiological Role and Pathogenic Potential in Cancer and Autoimmunity. Front Microbiol. 2018;9:462.

18. Brütting C, Narasimhan H, Hoffmann F, Kornhuber ME, Staege MS, Emmer A. Investigation of Endogenous Retrovirus Sequences in the Neighborhood of Genes Up-regulated in a Neuroblastoma Model after Treatment with Hypoxia-Mimetic Cobalt Chloride. Front Microbiol. 2018;9:287.

19. Machnik G, Skudrzyk E, Bułdak Ł, et al. Monitoring the Transcriptional Activity of Human Endogenous Retroviral HERV-W Family Using PNA Strand Invasion into Double-Stranded DNA. Mol Biotechnol. 2018;60(2):124-133.

20. Lu Q, Li J, Senkowski C, et al. Promoter Hypermethylation and Decreased Expression of Syncytin-1 in Pancreatic Adenocarcinomas. PLoS One. 2015;10(7):e0134412.

21. Huang Q, Chen H, Wang F, et al. Reduced syncytin-1 expression in choriocarcinoma BeWo cells activates the calpain1-AIF-mediated apoptosis, implication for preeclampsia. Cell Mol Life Sci. 2014;71(16): 3151-3164.

22. Soleymanlou N, Jurisicova A, Wu Y, et al. Hypoxic switch in mitochondrial myeloid cell leukemia factor-1/Mtd apoptotic rheostat contributes to human trophoblast cell death in preeclampsia. Am J Pathol. 2007;171(2):496-506.

23. Larsson LI, Bjerregaard B, Wulf-Andersen L, Talts JF. Syncytin and cancer cell fusions. Sci World J. 2007;7:1193-1197.

24. Larsen JM, Christensen IJ, Nielsen HJ, et al. Syncytin immunoreactivity in colorectal cancer: potential prognostic impact. Cancer Lett. 2009; 280(1):44-49.

25. Sun Y, Ouyang DY, Pang W, et al. Expression of syncytin in leukemia and lymphoma cells. Leuk Res. 2010;34(9):1195-1202.

26. Bergallo M, Daprà V, Calvi C, Montanari P, Galliano I, Ravanini P. Is HERV-K and HERV-W Expression Regulated by miR-155 in Kidney Transplant Patients with Human Cytomegalovirus Infection? Intervirology. 2018;61(1):23-29.

27. Strissel PL, Ruebner M, Thiel F, et al. Reactivation of codogenic endogenous retroviral (ERV) envelope genes in human endometrial carcinoma and prestages: Emergence of new molecular targets. Oncotarget. 2012;3(10):1204-1219.

28. Mo H, Ouyang D, Xu L, Gao Q, He X. Human endogenous retroviral syncytin exerts inhibitory effect on invasive phenotype of B16F10 melanoma cells. Chin J Cancer Res. 2013;25(5):556-564.

29. Curtin F, Bernard C, Levet S, et al. A new therapeutic approach for type 1 diabetes: Rationale for GNbAC1, an anti-HERV-WEnv monoclonal antibody. Diabetes Obes Metab. 2018;20(9): 2075-2084.

30. Bergallo M, Dapr V, Novelli M. Is HERV-K and HERV-W expression regulated by mir-155 in Sézary syndrome? G Ital Dermatol Venereol. Epub 2018 Mar 26. 
31. Dolei A. The aliens inside us: HERV-W endogenous retroviruses and multiple sclerosis. Mult Scler. 2018;24(1):42-47.

32. Arru G, Sechi E, Mariotto S, et al. Antibody response against HERV-W env surface peptides differentiates multiple sclerosis and neuromyelitis optica spectrum disorder. Mult Scler J Exp Transl Clin. 2017;3(4): 205521731774242.

33. Christensen T. Human endogenous retroviruses in the aetiology of MS. Acta Neurol Scand. 2017;136(Suppl 201):18-21.

34. Melbourne JK, Chase KA, Feiner B, Rosen C, Sharma RP. Long non-coding and endogenous retroviral RNA levels are associated with proinflammatory cytokine mRNA expression in peripheral blood cells: Implications for schizophrenia. Psychiatry Res. 2018;262: 465-468.

35. Tu X, Li S, Zhao L, Xiao R, Wang X, Zhu F. Human leukemia antigenA*0201-restricted epitopes of human endogenous retrovirus $\mathrm{W}$ family envelope (HERV-W env) induce strong cytotoxic T lymphocyte responses. Virol Sin. 2017;32(4):280-289.

36. Liu C, Liu L, Wang X, Liu Y, Wang M, Zhu F. HBV X Protein induces overexpression of HERV-W env through NF- $\mathrm{KB}$ in HepG2 cells. Virus Genes. 2017;53(6):797-806.

37. van Horssen J, van der Pol S, Nijland P, Amor S, Perron H. Human endogenous retrovirus $\mathrm{W}$ in brain lesions: Rationale for targeted therapy in multiple sclerosis. Mult Scler Relat Disord. 2016;8: $11-18$.

38. Stefanetti V, Marenzoni ML, Passamonti F, et al. High Expression of Endogenous Retroviral Envelope Gene in the Equine Fetal Part of the Placenta. PLoS One. 2016;11(5):e0155603.

39. Oppelt P, Strick R, Strissel PL, Winzierl K, Beckmann MW, Renner SP. Expression of the human endogenous retroviruse-W envelope gene syncytin in endometriosis lesions. Gynecol Endocrinol. 2009;25(11): $741-747$.
40. Morandi E, Tarlinton RE, Tanasescu R, Gran B. Human endogenous retroviruses and multiple sclerosis: Causation, association, or aftereffect? Mult Scler. 2017;23(8):1050-1055.

41. Bisogno LS, Friedersdorf MB, Keene JD. Ras Post-transcriptionally Enhances a Pre-malignantly Primed EMT to Promote Invasion. iScience. 2018:4:97-108.

42. Zhang W, Zhai Y, Wang W, Cao M, Ma C. Enhanced expression of lncRNA TP73-AS1 predicts unfavorable prognosis for gastric cancer and promotes cell migration and invasion by induction of EMT. Gene. 2018;678:377-383.

43. van de Merbel AF, van der Horst G, Buijs JT, van der Pluijm G. Protocols for Migration and Invasion Studies in Prostate Cancer. Methods Mol Biol. 2018;1786:67-79.

44. Liu L, Wu B, Cai H, et al. Tiam1 promotes thyroid carcinoma metastasis by modulating EMT via Wnt/ $\beta$-catenin signaling. Exp Cell Res. 2018; 362(2):532-540.

45. Hu Q, Yin J, Zeng A, et al. H19 functions as a competing endogenous RNA to regulate EMT by sponging miR-130a-3p in glioma. Cell Physiol Biochem. 2018;50(1):233-245.

46. Song N, Zhong J, Hu Q, et al. FGF18 enhances migration and the epithelial-mesenchymal transition in breast cancer by regulating Akt/GSK3 $\beta /$ B-Catenin signaling. Cell Physiol Biochem. 2018;49(3):1019-1032.

47. Burgess HM, Pourchet A, Hajdu CH, Chiriboga L, Frey AB, Mohr I. Targeting poxvirus decapping enzymes and mRNA decay to generate an effective oncolytic virus. Mol Ther Oncolytics. 2018;8:71-81.

48. Wang JH, Forterre AV, Zhao J, et al. Anti-HER2 scFv-directed extracellular vesicle-mediated mRNA-based gene delivery inhibits growth of HER2-positive human breast tumor xenografts by prodrug activation. Mol Cancer Ther. 2018;17(5):1133-1142.
OncoTargets and Therapy

\section{Publish your work in this journal}

OncoTargets and Therapy is an international, peer-reviewed, open access journal focusing on the pathological basis of all cancers, potential targets for therapy and treatment protocols employed to improve the management of cancer patients. The journal also focuses on the impact of management programs and new therapeutic agents and protocols on

\section{Dovepress}

patient perspectives such as quality of life, adherence and satisfaction The manuscript management system is completely online and includes a very quick and fair peer-review system, which is all easy to use. Visit http://www.dovepress.com/testimonials.php to read real quotes from published authors. 